\title{
6
}

\section{OCLUSÃO VASCULAR E SEUS EFEITOS SOBRE AS VIAS DE SINALIZAÇÃO DE HIPERTROFIA E ATROFIA MUSCULAR}

O treinamento resistido per se, estimula o crescimento muscular e diminui a atrofia pela ativação e inibição de vias de sinalização importantes (SPIERING et al., 2008). O mesmo também incita considerável resposta hormonal, como aumento dos níveis de hormônio de crescimento $(\mathrm{GH})$ e hormônio de crescimento tipo insulina (IGF-1) local e sistêmico que ativam as mesmas vias de hipertrofia supracitadas (SPIERING et al., 2008).

O treinamento resistido com oclusão vascular também demonstra resultados favoráveis sobre as vias de sinalização de hipertrofia e atrofia muscular. Fry et al. (2010) demonstraram que a síntese proteica pode ficar elevada em até 56\% quando o treinamento resistido de baixa intensidade (20\% 1RM) foi realizado com oclusão vascular. Além disso, aumentos da fosforilação de proteínas associadas a hipertrofia como S6K1, rpS6, ERK 1/2 e MnK1 que sugerem aumentos da sinalização da via mTOR (importante via para síntese proteica) após o exercício com oclusão vascular foram também identificados no estudo.

Dentre outras adaptações importantes para hipertrofia promovidas pelo treinamento resistido com o método oclusão vasclar, Wernbom et al. (2013) verificaram aumentos de 33-53\% de células satélites após o método oclusão vascular e Laurentino et al. (2002) verificaram que a expressão de genes da miostatina que é um importante regulador negativo dos processos de hipertrofia, diminuiu após 8 semanas de treinamento resistido com oclusão vascular, ao passo que, aumento 
dos níveis de força muscular, hipertrofia e gene da folistatina que é um importante supressor ou regulador fisiológico da miostatina foram verificados.

Isso representa uma grande aplicabilidade prática para o tratamento de síndromes do desuso, já que em períodos de destreinamento, existe uma correlação negativa entre o aumento dos níveis de miostatina e atrofia das fibras musculares tipo I e tipo II (JESPERSEN et al., 2011). Além do mais, elevaçōes dos níveis de miostatina estão presentes em doenças importantes como câncer, insuficiência cardíaca congestiva, AIDS, doença pulmonar crônica obstrutiva e sarcopenia (ARGILÉS et al., 2012).

O processo de envelhecimento com a sarcopenia que é a perda da massa muscular, está associado com aumentos dos riscos de quedas, fraturas e morte e dentre os biomarcadores utilizados para monitorar o tratamento da sarcopenia, a miostatina e folistatina representam duas importantes variáveis (WOO, 2017).

Considerando que o treinamento resistido com oclusão vascular tem demonstrado induzir hipertrofia com baixa carga (20\% 1RM) similar ao treinamento resistido de alta intensidade (PEARSON; HUSSAIN, 2015). Embora envolva baixa tensão mecânica, possui alto estresse metabólico que medeia o processo de hipertrofia pela produção sistêmica de hormônios (hormônio de crescimento) (TANIMOTO; MADARAME; ISHII, 2005), aumenta o recrutamento de fibras rápidas através da ativação da vias aferentes do tipo III e IV e inibição do neurônio motor alfa (YASUDA et al., 2010), induz inchaço celular, ativa vias de sinalização proteica, diminui a expressão de proteínas associadas a atrofia muscular (FOXO, miostatina, MuRF1 e MAFbx) (FRY et al., 2010; JESPERSEN et al., 2011; LAURENTINO et al., 2012; PEARSON; HUSSAIN, 2015), induz dano muscular (PEARSON; HUSSAIN, 2015), aumenta a difusão e proliferação de células satélites (PEARSON; HUSSAIN, 2015; WERNBOM et al., 2013), aumenta a quantidade de espécies reativas de oxigênio e expressão de genes da folistatina (LAURENTINO et al., 2011; PEARSON; HUSSAIN, 2015). Todas as alterações supracitadas são importantes para o processo de hipertrofia. A Tabela 1, adaptada de estudos anteriores (FRY et al., 2010; JESPERSEN et al., 2011; LAURENTINO et al., 2011; PEARSON; HUSSAIN, 2015; TANIMOTO; MADARAME; ISHII, 2005; WERNBOM et al., 2013; YASUDA et al., 2010) apresenta os possíveis efeitos do método oclusão vascular sobre as vias de hipertrofia e atrofia muscular. 
Tabela 1 Efeitos do método oclusão vascular sobre as vias de sinalização de hipertrofia e atrofia

\begin{tabular}{|ll|}
\hline \multicolumn{1}{|c|}{ Hipertrofia muscular } & \multicolumn{1}{c|}{ Atrofia muscular } \\
\hline Estresse metabólico $\uparrow$ & Fator de transcrição FOXO $\downarrow$ \\
\hline Lactato $\uparrow$ & Miostatina $\downarrow$ \\
\hline GH $\uparrow$ & MuRF1 $\downarrow$ \\
\hline IGF-1 $\uparrow$ & MAFbx $\downarrow$ \\
\hline Ativação de células satélites $\uparrow$ & Via da ubiquitina-proteassoma $\downarrow$ \\
\hline PI3K/Akt/mTOR $\uparrow$ & \\
\hline Recrutamento de fibras tipo II $\uparrow$ & \\
\hline Difusão e proliferação de células satélites $\uparrow$ & \\
\hline Interleucina- $6 \uparrow$ & \\
\hline Folistatina $\uparrow$ & \\
\hline
\end{tabular}

$\mathrm{GH}$ = hormônio de crescimento, IGF-1 = hormônio de crescimento tipo insulina, PI3K = fosfatidilinositol-3-quinase; $\mathrm{Akt}$ = proteína quinase $\mathrm{B}, \mathrm{mTOR}$ = alvo da rapamicina em mamíferos, $\mathrm{FOXO}$ = forkhead box $O, \mathrm{MuRF} 1=$ muscle RING-finger protein-1, MAFBx = muscle atrophy Fbox protein .

Com isso, um grande corpo de conhecimento tem demonstrado adaptações positivas do treinamento resistido de baixa intensidade realizado com oclusão vascular, sendo assim, muito importante nos processos de reabilitação muscular esquelética e sarcopenia (HUGHES et al., 2017). Lixandrão et al. (2018) em uma excelente revisão sistemática e meta-análise verificaram que o treinamento resistido de alta intensidade gerou ganhos de força muscular superiores quando comparado com o treinamento resistido com oclusão vascular. No entanto, os ganhos de massa magra foram similares entre os treinos.

Takarada et al. (2000) verificaram que o treinamento resistido de baixa intensidade (50\% 1RM) e com oclusão vascular quando comparado com o treinamento resistido de alta intensidade ( $80 \% 1 \mathrm{RM})$, após 16 semanas de treinamento em mulheres com idade média de 58 anos e saudáveis, induziu hipertrofia e força muscular similares quando comparado com o treinamento resistido de alta intensidade.

Considerando o baixo estresse mecânico e seu importante efeito em induzir hipertrofia e força muscular, o uso do método combinado com o treinamento resistido de baixa intensidade tem potencial efeito em acelerar o processo de recuperação muscular em pessoas idosas, ou diminuir o processo de atrofia muscular causado pela imobilização de membros após cirurgias ou internações (idosos acamados), afetando principalmente as vias de hipertrofia e atrofia muscular (BERG 
et al., 2007; BONALDO; SANDRI, 2013, 2013; KORTEBEIN et al., 2007). Idosos acamados, por exemplo, após 10 dias de imobilização diminuem cerca de $30 \%, 6 \%$ e $15 \%$ a taxa de síntese proteica, massa magra dos membros inferiores e força muscular, respectivamente (KORTEBEIN et al., 2007).

\section{REFERÊNCIAS BIBLIOGRÁFICAS}

ARGILÉS, Josep M. et al. Myostatin: more than just a regulator of muscle mass. Drug discovery today, v. 17, n. 13-14, p. 702-709, 2012.

BERG, Hans E. et al. Hip, thigh and calf muscle atrophy and bone loss after 5-week bedrest inactivity. European journal of applied physiology, v. 99, n. 3, p. 283-289, 2007.

BONALDO, Paolo; SANDRI, Marco. Cellular and molecular mechanisms of muscle atrophy. Disease models \& mechanisms, v. 6, n. 1, p. 25-39, 2013.

FRY, Christopher S. et al. Blood flow restriction exercise stimulates mTORC1 signaling and muscle protein synthesis in older men. Journal of applied physiology, v. 108, n. 5 , p. 1199-1209, 2010.

HUGHES, Luke et al. Blood flow restriction training in clinical musculoskeletal rehabilitation: a systematic review and meta-analysis. Br J Sports Med, v. 51, n. 13, p. 1003-1011, 2017.

JESPERSEN, J. G. et al. Myostatin expression during human muscle hypertrophy and subsequent atrophy: increased myostatin with detraining. Scandinavian journal of medicine \& science in sports, v. 21, n. 2, p. 215-223, 2011.

KORTEBEIN, Patrick et al. Effect of 10 days of bed rest on skeletal muscle in healthy older adults. Jama, v. 297, n. 16, p. 1769-1774, 2007.

LAURENTINO, Gilberto Candido et al. Strength training with blood flow restriction diminishes myostatin gene expression. Med Sci Sports Exerc, v. 44, n. 3, p. 406-12, 2012. LIXANDRĀO, Manoel E. et al. Magnitude of muscle strength and mass adaptations between high-load resistance training versus low-load resistance training associated with blood-flow restriction: A systematic review and meta-analysis. Sports Medicine, p. 1-18, 2017.

PEARSON, Stephen John; HUSSAIN, Syed Robiul. A review on the mechanisms of blood-flow restriction resistance training-induced muscle hypertrophy. Sports medicine, v. 45, n. 2, p. 187-200, 2015.

SPIERING, Barry A. et al. Resistance exercise biology. Sports Medicine, v. 38, n. 7, p. 527-540, 2008.

TAKARADA, Yudai et al. Effects of resistance exercise combined with moderate vascular occlusion on muscular function in humans. Journal of applied physiology, v. 88, n. 6, p. 2097-2106, 2000. 
TANIMOTO, M.; MADARAME, H.; ISHII, N. Muscle oxygenation and plasma growth hormone concentration during and after resistance exercise: Comparison between "KAATSU" and other types of regimen. International Journal of KAATSU Training Research, v. 1, n. 2, p. 51-56, 2005.

WERNBOM, Mathias et al. Acute low-load resistance exercise with and without blood flow restriction increased protein signalling and number of satellite cells in human skeletal muscle. European journal of applied physiology, v. 113, n. 12, p. 2953-2965, 2013. WOO, Jean. Sarcopenia. Clinics in geriatric medicine, v. 33, n. 3, p. 305-314, 2017. YASUDA, Tomohiro et al. Venous blood gas and metabolite response to low-intensity muscle contractions with external limb compression. Metabolism-Clinical and Experimental, v. 59, n. 10, p. 1510-1519, 2010. 
